\title{
EDUCACIÓN CON ADULTOS MAYORES: MOTIVACIONES Y BENEFICIOS PERCIBIDOS
}

\section{EDUCAÇÃO DA TERCEIRA IDADE: MOTIVAÇÕES E BENEFÍCIOS PERCEBIDOS}

\section{EDUCATION IN THE OLD AGE: PERCEPTION ON MOTIVATION AND BENEFITS}

\author{
Karina Valeria Dottori ${ }^{*}$ \\ Claudia Josefina Arias** \\ Corina Soliverez $z^{\star \star \star}$
}

\begin{abstract}
Resumen: El envejecimiento poblacional y la mejora en las condiciones de salud de los adultos mayores han traído como resultado nuevas demandas a las cuales dar respuesta. En este escenario, la educación ha tomado un rol protagónico para estos mayores activos y motivados para aprender y mejorar. Desde la Psicología Positiva y el enfoque del Curso de la Vida, se fortalece la idea del desarrollo y la obtención de ganancias en esta etapa de la vida, tradicionalmente asociada a pérdidas y deterioro. Desde su origen, las propuestas educativas para adultos mayores se han ampliado y diversificado en los últimos 40 años. En este sentido, el presente estudio propone por una parte, indagar sobre los beneficios percibidos frente a la participación en tales propuestas $(n=120)$ y, por otra, indagar los motivos por los que los adultos mayores eligen participar ( $n=60)$ o no de las mismas $(n=30)$. Se espera que los resultados obtenidos constituyan un aporte en el estudio de una temática reciente, considerada fundamental para el logro de un envejecimiento positivo.
\end{abstract}

Palabras clave: Adultos Mayores. Educación. Motivaciones. Beneficios percibidos.

\begin{abstract}
Population aging and improved health conditions for the elderly have resulted in new demands for which answers must be given. In this scenario, education has assumed a leading role for the elderly to remain active and motivated to learn and improve. From Positive Psychology to the focus of the Life Course, it strengths the idea that the development and achievements at this stage of life are traditionally associated with losses and deterioration. From its origin until the last 40 years, the educational proposals for the elderly have expanded and diversified. In this sense, the present study proposes, on the one hand, to question the perceived benefits of participation in such proposals $(n=120)$ and, on the other hand, to question the reasons why the older adults choose to participate $(n=60)$ or not $(n=30)$. It is hoped that the obtained results constitute a contribution to the study of a recent theme considered fundamental for the achievement of a positive aging.
\end{abstract}

Keywords: Old age. Education. Motivation. Benefits perceived.

Resumo: O envelhecimento populacional e a melhora das condições de saúde da terceira idade têm trazido como resultado novas demandas para as quais devem ser dadas respostas. Neste cenário, a educação tem assumido um protagonismo para que a terceira idade mantenha-se ativa e motivada a aprender e melhorar. Desde a Psicologia Positiva ao enfoque do Curso de Vida, fortalece-se a ideia de que o desenvolvimento e a obtenção de conquistas nesta etapa da vida são tradicionalmente associados

\footnotetext{
* Becaria de investigación de la Universidad Nacional de Mar del Plata, Buenos Aires, Argentina

** Investigadora de la Universidad Nacional de Mar del Plata, Buenos Aires, Argentina

*** Docente da Universidad Nacional de Mar del Plata. E-mail: csoliverez@yahoo.com.ar
} 
a perdas e deterioração. Deste sua origem até os últimos 40 anos, as propostas educativas para a terceira idade tem se ampliado e diversificado. Neste sentido, o presente estudo propõe, de um lado, quiestionar os benefícios percebidos frente à participação em tais propostas $(n=120)$ e, de outro, questionar os motivos pelos quais a terceira idade escolhe participar $(n=60)$, ou náo, das mesmas $(n=30)$. Espera-se que os resultados obtidos constituam um aporte para o estudo de uma temática recente, considerada fundamental para o alcance de um envelhecimento positivo.

Palavras-chave: Terceira idade. Educação. Motivações. Benefícios percebidos.

\section{Introducción}

En el contexto actual, reflexionar sobre el envejecimiento, implica remitirse a una gran variedad de situaciones, investigaciones y conceptos que evidencian la heterogeneidad característica de esta etapa vital, así como la coexistencia de estereotipos y representaciones sociales diferentes.

Asimismo, implica situarse en el ya conocido proceso de envejecimiento poblacional que se profundiza constantemente a nivel mundial. De acuerdo con el Informe de Estado de la Población Mundial (2011), realizado por el Fondo de Población de las Naciones Unidas (UNFPA), en América Latina la esperanza de vida ha aumentado casi 20 años en el transcurso de las últimas cinco décadas, y específicamente en el caso de Argentina los estudios demográficos muestran que es uno de los países más envejecidos de la región junto con Uruguay, Cuba y Chile.

Si bien persisten estereotipos que consideran a la vejez como una etapa de pérdidas y deterioro, distintos enfoques teóricos y hallazgos científicos permiten visibilizar al envejecimiento como un proceso en donde también suceden ganancias. Recientemente, dentro del campo de la Gerontología y de la Psicología de la vejez, se está incluyendo el estudio de fortalezas, habilidades y competencias, que permiten una mayor comprensión de la variabilidad y complejidad del proceso de envejecimiento humano (ARIAS; IACUB, 2013; SELIGMAN,
2002; SELIGMAN; CSIKSZENTMIHALYI, 2000; BALTES, REESE; LIPSITT, 1980).

Estos estudios han demostrado que gran parte de los adultos mayores, además de tener una mayor expectativa de vida, poseen niveles educativos más elevados, han logrado más autonomía y libertad, envejecen con mejores condiciones de salud y una mayor independencia económica, disponen de más tiempo y muestran gran interés por adquirir nuevos conocimientos y destrezas (PINAZO, et all., 2010).

En el marco del desarrollo a lo largo de la vida y como respuesta a las nuevas demandas de los adultos mayores, se han propuesto y puesto en marcha variadas prácticas orientadas a mejorar su bienestar, promover su participación social y potenciar los recursos de los que disponen (ARIAS, 2015).

\section{Talleres socioeducativos: una alternativa de gran impacto y desarrollo}

Una de las primeras prácticas orientadas a promover la participación e incrementar el bienestar de los adultos mayores han sido los talleres socioeducativos: propuestas grupales e inclusivas, gratuitas o con aranceles bajos, cuyo único requisito para participar es poseer un mínimo de edad establecido y que abarcan temáticas recreativas, culturales, intelectuales, físicas o comunitarias, entre otras. 
Desde el enfoque de la educación a lo largo de la vida, se ha resaltado el derecho de los adultos mayores a acceder a la misma. Asimismo, el tema ha ganado un espacio importante en declaraciones y asambleas internacionales sobre los derechos de las personas mayores, y ha llegado incluso al ámbito académico, delineando investigaciones sobre la importancia, motivos y beneficios de participar en actividades educativas (DOTTORI, 2015).

La oferta de talleres y de cursos para adultos mayores desde diferentes organismos públicos y privados no sólo ha crecido en número, sino que también se ha diversificado. Esta situación se observa en una creciente cantidad de países, abarcando una gran variedad de temáticas que brindan amplias posibilidades para el desarrollo intelectual, físico, personal y social.

En Argentina también se observa la evolución de los talleres tanto a nivel cuantitativo como cualitativo. El número de inscriptos ha aumentado notablemente desde sus inicios hasta la actualidad, encontrándose propuestas que responden a temáticas diversas ofrecidas por organismos públicos y privados (SOLIVEREZ; DOTTORI, 2013; YUNI, 2011).

En la última década, con el objetivo de profundizar el estudio y medir el impacto de estas propuestas, diversos investigadores se han abocado a evaluar los beneficios percibidos por los propios adultos mayores, así como los motivos que los llevan a participar. Los resultados indicaron que involucrarse en este tipo de actividades: aumenta el bienestar, favorece una autopercepción positiva del envejecimiento y el balance emocional (Fernández-Ballesteros et. al., 2013), mejora la percepción de la calidad de vida y la autovaloración (Villar et. al., 2006) y favorece la integración social a través del contacto con otros adultos mayores y jóvenes (MONTORO; PINAZZO, 2005; YUNI, URBANO; TARDITI, 2012).

Villar et al (2010) encontraron que los beneficios percibidos estaban ligados a aspectos sociales y anímicos, ganar amigos, aumentar la satisfacción con la vida y alegría de vivir. Los motivos más frecuentes eran de índole cognitivo y expresivo: adquirir conocimientos, abrir la mente, y aprender por el placer que esto les proporcionaba.

Según Montoro, Pinazo y Tortosa (2007), las personas mayores participan en este tipo de propuestas por diversos motivos que incluyen en general de una manera combinada intereses de tipo cognitivo, búsqueda de contacto social y generación de nuevos vínculos, satisfacción y diversión.

Puede observarse que tanto los beneficios percibidos como los motivos para participar son múltiples, heterogéneos, y se presentan de manera combinada. Asimismo, los aportes de investigación en torno a estos aspectos datan de hace pocos años, y no se cuenta con información acerca de un grupo importante de adultos mayores que no accede a estas propuestas. Por este motivo, el estudio que aquí se presenta se propuso indagar los beneficios percibidos por adultos mayores de la ciudad de Mar del Plata, Argentina, que participan en talleres y cursos para adultos mayores y, en un segundo momento, explorar tanto los motivos que los llevan a participar en actividades formativas como las razones por las que algunos adultos mayores deciden no participar de estas propuestas. Los datos de este estudio constituyen un aporte novedoso y local a una temática reciente, considerada fundamental para el logro de un envejecimiento positivo.

\section{Metodología}

Se implementó un diseño de tipo no experimental, transversal, descriptivo. 
El muestreo fue realizado de forma no probabilística intencional, y en todos los casos se administró un cuestionario de datos sociodemográficos. Mediante consentimiento informado escrito fue explicitado el carácter voluntario de la participación, la confidencialidad de la información de acuerdo con la Ley Nacional 25.326 de protección de los datos personales y la finalidad científicoacadémica de los resultados obtenidos.

El estudio se realizó en dos etapas: en un primer momento, se buscó evaluar el impacto de los Talleres socio-educativos para Adultos Mayores de la ciudad de Mar del Plata en las áreas psicológica, física y social. Para ello, se seleccionó una muestra de 120 adultos mayores de ambos sexos que poseían 60 años o más de edad y que hubieran participado o estuvieran participando en Talleres para Adultos Mayores en la ciudad de Mar del Plata. Se les administró un Cuestionario de preguntas cerradas elaborado por Lacub y Arias (2012), que consta de 77 ítems y que explora el impacto de la participación en los talleres en diversas áreas: psicológica, física y social. Todos los ítems tienen tres opciones de respuesta que permiten comparar la situación posterior a la participación en los talleres con la anterior a la misma. Por esta razón, la persona puede responder si su situación en los distintos aspectos evaluados ha mejorado, se ha mantenido igual o ha disminuido.

En una segunda etapa, se buscó explorar las razones que motivan la participación o no de adultos mayores residentes en la Ciudad de Mar del Plata en los mencionados talleres. En este caso, la muestra estuvo conformada por 60 adultos mayores que participaban en talleres y 30 adultos mayores que no participaban. Al grupo participante se le administró un cuestionario de motivos para realizar actividades formativas (Solé et. al., 2005). El mismo es una escala Likert de 17 ítems que evalúa motivos para incluirse en propuestas formativas en términos de "nada”, "poco", "bastante" o "mucho" y que, de acuerdo al estudio citado, posee propiedades psicométricas adecuadas. Finalmente, con el grupo no participante se realizó una breve entrevista semi-dirigida que explora los motivos por los cuales no se incluyen en talleres para mayores.

Los datos obtenidos fueron analizados a través de paquetes informatizados para su uso en Ciencias Sociales, utilizando técnicas de estadística descriptiva.

\section{Resultados}

Los resultados de la primera etapa indicaron que a partir de la participación en los talleres, los cambios positivos informados más frecuentes fueron los referidos a aspectos sociales, encontrándose de manera frecuente, pero en menor cantidad, cambios a nivel psicológico y no hallando frecuencias elevadas en ningún aspecto del área salud física.

Como puede observarse en la Tabla $\mathrm{n}^{0} 1^{1}$, entre el $80 \%$ y el $90 \%$ de los participantes tiene más interés en emprender actividades, se siente más eficaz en el logro de los objetivos propuestos, tiene mayor voluntad para realizar nuevas actividades, realiza más actividades que antes y considera que ha mejorado su capacidad para transmitir y compartir su conocimiento. A su vez, entre el 50\% y el 75\% refirió tener más temas para compartir con su familia, haber mejorado su participación social, tener más encuentros con amistades y sumarse a otras propuestas para adultos mayores con más frecuencia que antes.

En cuanto a los aspectos psicológicos, más de las tres cuartas partes de los entrevistados consideraron que mejoró su

${ }^{1}$ El sombreado indica los cambios positivos que poseen las frecuencias más elevadas (>70\%). 
capacidad intelectual, que obtuvieron más conocimientos sobre la vejez y que mejoró su estado de ánimo. Asimismo, más de la mitad expresó que había mejorado su bienestar general, que se encontraban más entusiasmados y alegres, que había mejorado su idea acerca de la vejez, que habían obtenido nuevos recursos para desarrollar un proyecto personal, que se valoraban más que antes, que percibían que los demás los veían mejor, que habían mejorado su idea sobre las otras personas mayores, y que se sentían más tolerantes con la gente que pensaba de un modo diferente a ellos. También expresaron haber notado cambios positivos en otros aspectos relativos a la capacidad intelectual como la rapidez para pensar, la capacidad de comprensión, la atención y la expresión en palabras.

Tabla 1: Cambios positivos observados en la mayoría de los adultos mayores encuestados

\begin{tabular}{|c|c|c|c|}
\hline \multirow{2}{*}{ 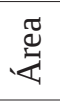 } & \multirow{2}{*}{ Ítem } & \multicolumn{2}{|c|}{ Hubo cambios positivos } \\
\hline & & $\mathrm{N}^{\circ}$ & $\%$ \\
\hline \multirow{9}{*}{ 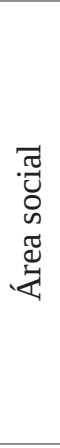 } & Interés en emprender actividades & 109 & 90,8 \\
\hline & Sentimiento de eficacia en el logro de objetivos & 99 & 82,5 \\
\hline & Voluntad para realizar actividades & 99 & 82,5 \\
\hline & Cantidad de actividades que realiza & 98 & 81,7 \\
\hline & Capacidad para transmitir y compartir su conocimiento & 96 & 80,0 \\
\hline & Cantidad de temas p/compartir con la familia & 87 & 72,5 \\
\hline & Participación social & 82 & 68,3 \\
\hline & Cantidad de encuentros con amistades & 69 & 57,5 \\
\hline & Se suma a otras propuestas para AM & 68 & 56,7 \\
\hline \multirow{18}{*}{ 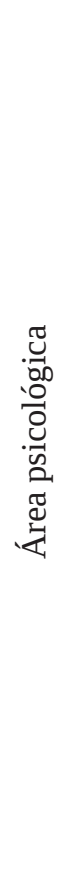 } & Capacidad Intelectual & 96 & 80,0 \\
\hline & Cantidad de conocimientos sobre la vejez & 96 & 80,0 \\
\hline & Estado de entusiasmo & 90 & 75,0 \\
\hline & Estado de ánimo & 84 & 70,0 \\
\hline & Bienestar general & 82 & 68,3 \\
\hline & Capacidad para aprender & 77 & 64,1 \\
\hline & Recursos para desarrollar un proyecto personal & 77 & 64,1 \\
\hline & Estado de alegría & 76 & 63,3 \\
\hline & Idea o concepto de la vejez & 76 & 63,3 \\
\hline & Idea sobre otras personas mayores & 74 & 61,7 \\
\hline & Tolerancia hacia gente que piensa distinto & 62 & 61,7 \\
\hline & Rapidez para pensar & 73 & 60,8 \\
\hline & Capacidad de comprensión & 73 & 60,8 \\
\hline & Capacidad para expresarse en palabras & 72 & 60,0 \\
\hline & Percepción de cómo lo ven los demás & 72 & 60,0 \\
\hline & Capacidad para prestar atención & 72 & 60,0 \\
\hline & Autovaloración & 70 & 58,3 \\
\hline & Memoria & 68 & 56,7 \\
\hline
\end{tabular}


Cabe destacar que si bien no se han detectado cambios positivos frecuentes en ningún aspecto del área salud física, si se realiza un análisis por área se observa que casi el 40\% de los participantes encontraron al menos un cambio positivo, vinculado principalmente con una mejora en la auto-percepción de la salud y una disminución en la frecuencia de los dolores corporales (ver Tabla ${ }^{\circ} 2$ ).

Tabla 2: Percepción de cambios en el área salud física

\begin{tabular}{c|c|c|c|c|c|c}
\hline & \multicolumn{2}{|c|}{$\begin{array}{c}\text { Hubo } \\
\text { cambios } \\
\text { positivos }\end{array}$} & \multicolumn{2}{|c|}{$\begin{array}{c}\text { No hubo } \\
\text { cambios } \\
\text { positivos }\end{array}$} & \multicolumn{2}{|c}{ Total } \\
\cline { 2 - 7 } & $\mathrm{N}^{\mathrm{o}}$ & $\%$ & $\mathrm{~N}^{\circ}$ & $\%$ & $\mathrm{~N}^{\circ}$ & $\%$ \\
\hline $\begin{array}{c}\text { Área } \\
\text { física }\end{array}$ & 44 & 36,7 & 76 & 73,3 & 120 & 100,0 \\
\hline
\end{tabular}

En cuanto a las diferencias por género, los resultados mostraron que en ningún caso se presentaban diferencias estadísticamente significativas entre varones y mujeres (prueba de $x^{2}$ ).

En una segunda etapa, se exploraron las razones que motivan la inclusión o no inclusión de adultos mayores residentes en la Ciudad de Mar del Plata en Talleres para adultos mayores.

En ambos casos, los resultados fueron múltiples y diversos. Dentro del grupo participante, los motivos aducidos con mayor frecuencia son los que se refieren a una búsqueda intelectual o personal. En este sentido, más del $90 \%$ consideró como un motivo bastante importante o muy importante "estar activo mentalmente" y "crecer como persona”. Asimismo, entre un 70\% y un 89\% consideró bastante importante o muy importante motivos de orden anímico y vocacional, como "pasarlo bien”, “distraerse”, “vocación o afición”, razones referidas a la ocupación del tiempo libre, al establecimiento de vínculos y a la mejora de la salud. El motivo menos frecuente fue "para no estar solo" (ver tabla n³). En cuanto a las razones que llevan a los adultos mayores a no participar, las más frecuentes fueron el desconocimiento y el desinterés. Asu vez, cerca del 30\% se refirió a no tener tiempo por estar trabajando o manifestó una idea vaga o errónea sobre el contenido de los talleres. Otros motivos aducidos con menor frecuencia fueron la falta de tiempo por tener que cuidar a un familiar dependiente (hijos, padres) o por estar realizando otras actividades, cuestiones personales como la falta de constancia o no querer comprometerse, cuestiones relativas a la edad (“estar viejo”) y depender de un familiar por tener dificultades físicas y/o económicas (ver tabla n4).

Tabla 3: Frecuencias y porcentajes de motivos para realizar actividades formativas*

\section{Motivos para realizar actividades formativas}

\begin{tabular}{l|c|c}
\hline \multirow{2}{*}{} & \multicolumn{2}{|c}{$\begin{array}{c}\text { Bastante/ } \\
\text { Mucho }\end{array}$} \\
\cline { 2 - 3 } & $\mathrm{N}^{\circ}$ & $\%$ \\
\hline Estar activo/a mentalmente & 55 & 91,7 \\
\hline Crecer como persona & 55 & 91,6 \\
\hline Pasarlo bien & 53 & 88,4 \\
\hline Ocupar el tiempo de manera útil & 52 & 86,6 \\
\hline Profundizar conocimientos & 52 & 86,6 \\
\hline Vocación o afición & 49 & 81,7 \\
\hline Conocer gente & 47 & 78,4 \\
\hline Distraerse & 45 & 75,0 \\
\hline Compartir momentos de ocio & 43 & 71,7 \\
\hline Mejorar la salud & 42 & 70,0 \\
\hline Pertenencia a un grupo de la misma edad & 37 & 61,7 \\
\hline Obligación de salir de casa & 35 & 58,4 \\
\hline Compartir trabajo voluntario & 34 & 56,7 \\
\hline No pensar en los problemas & 29 & 48,3 \\
\hline Relación con personas jóvenes & 28 & 46,7 \\
\hline Solución de problemas cotidianos & 26 & 43,3 \\
\hline Para no estar solo/a & 18 & 30,0 \\
\hline
\end{tabular}

* El sombreado más oscuro indica los motivos que poseen las frecuencias más elevadas (>90\%), mientras que el sombreado más claro indica los motivos que poseen una frecuencia de entre el $70 \%$ y el $89 \%$. En la frecuencia atribuida a cada motivo $\left(\mathrm{N}^{\circ}\right)$ se consideró la suma de quienes refirieron a la importancia de ese motivo como "bastante” y "mucho". 
Tabla 4: Frecuencias y porcentajes de motivos por los cuales los adultos mayores no participan en talleres ${ }^{*}$

\begin{tabular}{l|c|c}
\hline \multicolumn{3}{c}{$\begin{array}{c}\text { Motivos por los cuales los adultos mayores } \\
\text { no participan en talleres }\end{array}$} \\
\hline & $\begin{array}{c}\text { Bastante/ } \\
\text { Mucho }\end{array}$ \\
\hline & $\mathrm{N}^{\mathrm{o}}$ & $\%$ \\
\hline Desconocimiento & 16 & 53,3 \\
\hline Desinterés & 14 & 46,6 \\
\hline Idea vaga o errónea & 8 & 26,6 \\
\hline Falta de tiempo por estar trabajando & 8 & 26,6 \\
\hline $\begin{array}{l}\text { Falta de tiempo por tener que } \\
\text { cuidar un familiar dependiente } \\
\text { (hijos, padres) }\end{array}$ & 5 & 16,6 \\
\hline Falta de constancia & & 13,3 \\
\hline "Ya estoy viejo" & 4 & 13,3 \\
\hline No querer comprometerse & 4 & 13,3 \\
\hline $\begin{array}{l}\text { Falta de tiempo por estar realizando } \\
\text { otras actividades }\end{array}$ & 3 & 10,0 \\
\hline $\begin{array}{l}\text { Dependen de un familiar por } \\
\text { dificultades físicas y/o económicas }\end{array}$ & 2 & 06,6 \\
\hline
\end{tabular}

${ }^{*}$ El sombreado indica los motivos más frecuentes. En la frecuencia atribuida a cada motivo $\left(\mathrm{n}^{\circ}\right)$ se consideró la suma de quienes refirieron a la importancia de ese motivo como "bastante" y "mucho".

\section{Discusión}

Los resultados obtenidos son congruentes con los aportes de la Psicología Positiva y del enfoque del Curso de la Vida. Los hallazgos fortalecen empíricamente la idea de que los Adultos Mayores pueden seguir aprendiendo y potenciando sus capacidades a nivel psicológico y a nivel social, además, la importancia de los talleres para adultos mayores como espacios apropiados para el desarrollo de estas capacidades.

Como puede observarse, los resultados obtenidos en relación a los beneficios percibidos atribuidos a la participación en talleres son similares a los logrados en investigaciones anteriores, sobre todo en lo relativo a la mejora en los vínculos y, dentro del área psicológica, a una mejora en el estado de ánimo, la autovaloración, y el desarrollo de aspectos cognitivos. Con respecto a los cambios percibidos en el área física, en la mayoría de los casos no se lo atribuye a la participación en los talleres. Sin embargo, es posible observar que algunos participantes perciben al menos un cambio positivo en esta área, por lo cual podría pensarse en nuevas formas de indagación que permitan una búsqueda más amplia y profunda.

Con respecto a los motivos que llevan a incluirse en talleres, se observa que estos son múltiples y variados, destacándose motivos intelectuales y de desarrollo personal, en coincidencia con lo obtenido en investigaciones previas. En cuanto a las razones por las que no participan, si bien toman un lugar preponderante el desconocimiento o el desinterés, es notable observar la variedad de motivos que - aunque con una frecuencia menor - también estarían influyendo: la falta de tiempo por tener que cumplir el rol de cuidadores o de encontrarse trabajando aún, ciertas situaciones de dependencia por motivos económicos o de salud, la valoración de uno mismo por considerarse inconstante o por considerarse "viejo" y decisiones personales como no querer comprometerse.

Esta multiplicidad y diversificación de motivos que llevan a participar o no participar en tales propuestas confirma la heterogeneidad de este colectivo social. Esto, a su vez, reafirma la necesidad de pensar estrategias que den cuenta de dicha heterogeneidad y favorezcan su acercamiento a estas propuestas, incluyendo una mayor cantidad de hombres en las aulas y personas de distintos niveles socioeconómicos y educativos. 
En cuanto a las limitaciones del estudio, por tratarse de resultados obtenidos a partir de una muestra intencional las conclusiones no pueden generalizarse al total de la población. Asimismo, cabe destacar que las personas que concurren a estos espacios y que como consecuencia de esto han formado parte de la muestra, poseen un marcado sesgo de nivel educativo medio-alto y son auto-válidos, mientras que las personas que no concurren a talleres tenían en su mayoría un nivel educativo medio-bajo. Estas características, aunque coincidentes con las halladas por otros investigadores en sus estudios empíricos, podrían incidir en los resultados alcanzados.

En investigaciones posteriores se podría profundizar la temática con metodologías cualitativas y ampliar la muestra de personas que no participan para pensar alternativas de inclusión, indagando no solo acerca de los motivos, sino acerca de posibles intereses o necesidades.

Finalmente, se considera como fortaleza de este estudio haber realizado un aporte actual a nivel local, desde la perspectiva de los propios adultos mayores, sobre una temática de relevancia para el logro de un envejecimiento positivo. Se espera que los resultados obtenidos acerca de los beneficios den sustento empírico a estas propuestas al evidenciar su impacto, y que la información acerca de los motivos que llevan a participar o no participar pueda ser de utilidad a la hora de pensar el diseño y oferta de nuevas propuestas.

\section{Consideraciones finales}

El aprendizaje y la participación social son necesidades de estos nuevos adultos mayores, los cuales se encuentran más motivados, en mejor estado de salud y resultan más longevos que en décadas anteriores, y que, por lo tanto, plantean demandas a las que hay que continuar dando respuesta. Sus motivaciones y condiciones sociales, económicas, familiares y culturales son sumamente heterogéneas, por lo cual es complejo, aunque necesario, pensar en propuestas que puedan responder a todos sus intereses y necesidades.

En todo caso, la cuestión es cómo garantizar que las posibilidades educativas a lo largo de la vida - entendidas como un derecho social - se cumplan. Esta tarea implica un rol activo por parte de los gobiernos y la articulación público-privada con los distintos actores involucrados. Las universidades deben promocionar la educación e innovar en metodologías y temáticas, además de investigar y entregar información que ayude a la generación de políticas y propuestas (Rojas, 2012).

\section{Referencias bibliográficas}

ARIAS, C. Investigación y programas sociales: prácticas gerontológicas positivas y reconstrucción de vínculos. In: Conectando la ciencia con la sabiduría de nuestros viejos pueblos. La Paz: Universidad Mayor de San Andres/Redip, 2015, p.123-131.

ARIAS, C.; IACUB, R. ¿Por qué investigar aspectos positivos enlavejez? Contribuciones para un cambio de paradigma. Publicatio UEPG Ciencias Humanas, Lingüística, Letras e Artes, v.21, n.2, 271-281, 2013.

BALTES, P.; REESE, H.; LIPSITT, L. Lifes pandevelop mental psychology. Annual Review of Psychology, v.31, 65-110, 1980.

BERMEJO, L. Pedagogía gerontológica y buenas prácticas socioeducativas con personas mayores. En: BERMEJO, L. Envejecimiento activo $y$ actividades socioeducativas con personas mayores. 
Madrid: Editorial Medica Panamericana, 2010, p.11-34.

DOTTORI, K. Talleres socioeducativos. Kairos. 2015.

FERNÁNDEZ-BALLESTEROS, R.et. al. Effects of university programs for older adults: changes in cultural and group stereotype, self-perception of aging, and emotional balance. Educational Gerontology, vol.39, n², 119-131, 2013.

IACUB, R.; ARIAS, C. Cuestionario de exploración de cambios físicos, psicológicos y sociales a partir de la participación en talleres. Buenos Aires. 2012.

MONTORO, J.; PINAZO, S. Evaluating socialintegrationandpsychologicaloutcomes for older adults enrolled at a university intergenerational program. Journal of Intergenerational Relationships, vol.3, n³, 63-80, 2005.

MONTORO, J.; PINAZO, S.; TORTOSA, M. Motivaciones y expectativas de los estudiantes mayores de 55 años en los programas universitarios. Revista Sociedad Española de Geriatría y Gerontología, vol.42, 158-166, 2007.

PINAZO, X. et. al. Envejecimiento activo y aprendizaje a lo largo de la vida. In: BERMEJO, L. Envejecimiento activo y actividades socioeducativas con personas mayores. Madrid: Editorial Médica Panamericana, 2010, p.3-10.

ROJAS, M. Educación y capacitación para personas mayores: lo que existe y lo que se requiere. Aportes desde la visión de la universidad. En: Cuarta Conferencia Internacional Oxford Institute of Ageing LARNA. Actas... Santiago de Chile: Pontífica Universidad Católica de Chile. 2012.
SELIGMAN, M. (2002). La auténtica felicidad. Barcelona: Vergara, 2003. 428p.

SELIGMAN, M.; CSIKSZENTMIHALYI, M. Positive psychology: an Iintroduction. American Psychologist, v.55, n.1, 5-14, 2000.

SOLÉ, C.et. all. La educación en la vejez: razones para participar en programas educativos y beneficios que se extraen. Revista de Ciencias de la Educación, vol. 203, 453-465, 2005.

SOLIVEREZ, C.; DOTTORI, K. Propuestas positivas para adultos mayores: avances y desarrollos. In: La psicología en la sociedad contemporánea: actualizaciones, problemáticas y desafíos, $3^{\circ}$ Congreso de Psicología del Tucumán. Actas...Facultad de Psicología: Universidad Nacional del Tucumán. 2013.

UNFPA. Fondo de Población de las Naciones Unidas. Estado de la población mundial 2011. Disponible en: <https:// www.unfpa.org/sites/default/files/pub-pdf/ SP-SWOP2011_Final.pdf $>$. Acceso en: 24 nov. 2015.

VILLAR POSADA, F. (Convocatoria 2006). Evaluación de programas universitarios para mayores: motivaciones, dificultades y contribuciones a la calidad de vida demanda y prestación de cuidados en el seno familiar. Madrid: Estudios de I+D+I, n50, 2010. Disponible en: <http://envejecimiento. csic.es/documentos/ documentos/imsersoestudiosidi-50.pdf $>$. Acceso en: 25 nov. 2015.

VILLAR, F; et. al. Older people’s university students in Spain: a comparison of motives and benefits between two models. Ageing and Society, vol.30, nº, 1357-1372, 2010.

YUNI, J. Recorridos, limitaciones y posibilidades de las experiencias de 
educación no formal universitaria en argentina. En: IV Congreso Iberoamericano de Universidades para Mayores. Actas. Alicante: Asociación Estatal de Programas Universitarios para Mayores. 2011.

YUNI, J.; URBANO, C.; TARDITI, L. La educación como recurso para la integración social de los adultos mayores. 2012. Disponible en: <http://www. redadultosmayores.com.ar/buscador/files/ EDUCA024_YuniTarditi.pdf $>$. Acceso en: 25 nov. 2015. 